\title{
THE LEAST NUMBER OF $n$-PERIODIC POINTS ON TORI CAN BE REALIZED BY A SMOOTH MAP
}

\author{
JERZY JEZIERSKI
}

\begin{abstract}
We give an algebraic proof of the Theorem of Cheng Ye You that the least number of $n$-periodic points, in the continuous homotopy class of a self-map of a torus, can be realized by a smooth map.
\end{abstract}

\section{Introduction}

The classical Nielsen number $N(f)$, of a continuous self-map of a compact polyhedron $f: X \rightarrow X$, is a homotopy invariant and a lower bound of fixed points in the homotopy class: i.e. for each $g$ homotopic to $f$ the inequality $\# \operatorname{Fix}(g) \geq$ $N(f)$ holds. This number was generalized by Boju Jiang to a homotopy invariant lower bound of the number of $n$-periodic points: \#Fix $\left(g^{n}\right) \geq N F_{n}(f)$ for each $g$ homotopic to the given $f[17]$. Later it was generalized to $N J D_{n}(f)$, a lower bound of the number of $n$-periodic points in the smooth homotopy class of a selfmap $f: M \rightarrow M$ of a compact manifold [7]. The obvious inequality $N F_{n}(f) \leq$ $N J D_{n}(f)$ often turns out to be sharp [6]. However Cheng Ye You had proved much earlier that in the case of self-maps of a torus the both numbers coincide [20]. This result easily generalizes to all self-maps of nil- and solvmanifolds [14]. The proofs, in [20] and in [14], are geometric and they do not use the above invariants. On the other hand the generalized Nielsen theory was successfully used to show when a similar equality takes place in the case of self-maps of

2010 Mathematics Subject Classification. Primary: 55M20; Secondary: 54H25, 57R99.

Key words and phrases. Fixed point; periodic point; Nielsen fixed point theory; Dold congruences; least number of periodic points. 
compact Lie groups with free fundamental group [15]. Here we will reprove the Theorem of Cheng Ye You for self-maps of tori: we will show that an algebraic condition for the equality is satisfied for self-maps of the torus. In a forthcoming paper we will use this condition to prove when the equality holds for any Lie groups. Since in Nielsen theory simple algebraic methods are effective only in dimensions $\geq 3$ (Wecken Theorem), in the last Section we give elementary proof of this Theorem in low dimensions. Of course the last proof must be again geometric.

In the first Sections we recall basic information on Nielsen periodic point theory. In Sections 4 and 5 we recall conditions for smooth realizability of the number of periodic points: Lemma 4.2 and Theorem 5.2. In Section 6 we show that these conditions are satisfied for all self-maps of a torus of dimension $\geq 3$. In the last Section we consider the low dimensional cases.

Here by smoothness we mean $C^{1}$, although use of any other class $C^{\alpha}$, where $\alpha=1, \ldots, \infty$, leads to the same results, since the sequence of indices $\left(\operatorname{ind}\left(f^{k} ; x_{0}\right)\right)$ realizable by a $C^{1}$ map is also realizable by a $C^{\infty}$ map [8].

\section{Nielsen fixed and periodic points theory}

For the details we send the reader to [17] and [16]. We consider a self-map of a compact connected polyhedron $f: X \rightarrow X$ and its fixed point set $\operatorname{Fix}(f)$. We define the Nielsen relation on this set by: $x \sim y$ if and only if there is a path $\omega$ joining $x$ with $y$ so that $f \omega$ and $\omega$ are fixed end point homotopic.

This relation splits $\operatorname{Fix}(f)$ into Nielsen classes. Their set will be denoted by $\mathcal{N}(f)$. We say that a Nielsen class $A$ is essential if its fixed point index is nonzero: $\operatorname{ind}(f ; A) \neq 0$. The number of essential Nielsen classes is called Nielsen number and is denoted $N(f)$. This is a homotopy invariant and moreover it is the lower bound of the number of fixed points in the (continuous) homotopy class: $N(f) \leq \min _{h \sim f} \# \operatorname{Fix}(h)[3],[17],[16]$.

On the other hand we define, the set of Reidemeister classes of the map $f$ as the quotient set of the action of the fundamental group $\pi_{1} M$ on itself given by $\omega * \alpha=\omega \cdot \alpha \cdot\left(f_{\#} \omega\right)^{-1}$. Here we take as the base point a fixed point $x_{0} \in \operatorname{Fix}(f)$. We denote the quotient space by $\mathcal{R}(f)$. There is a natural injection from the set of the Nielsen classes to the set of Reidemeister classes $\mathcal{N}(f) \subset \mathcal{R}(f)$ defined as follows. We choose a point $x$ in the given Nielsen class $A$ and a path $\omega$ from the base point $x_{0}$ to $x$. Then the loop $\omega *(f \omega)^{-1}$ represents the corresponding Reidemeister class.

We will denote the Reidemeister classes of iterations as $[\alpha]^{n} \in \mathcal{R}\left(f^{n}\right)$ where $\alpha \in \pi_{1} M$.

Now we consider iterations of the map $f$. For fixed natural numbers $l \mid k$ there is a natural inclusion $\operatorname{Fix}\left(f^{l}\right) \subset \operatorname{Fix}\left(f^{k}\right)$ which induces the map $\mathcal{N}\left(f^{l}\right) \rightarrow \mathcal{N}\left(f^{k}\right)$ 
(which may be not injective). This map extends to $i_{k l}: \mathcal{R}\left(f^{l}\right) \rightarrow \mathcal{R}\left(f^{k}\right)$ and the last is given by

$$
i_{k l}[\alpha]^{l}=\left[\alpha \cdot f^{l}(\alpha) \cdot f^{2 l}(\alpha) \cdot \ldots \cdot f^{k-l}(\alpha)\right]^{l} .
$$

The functorial equalities $\left(i_{k l} i_{l m}=i_{k m}, i_{k k}=\right.$ id $)$ are satisfied and moreover the diagram

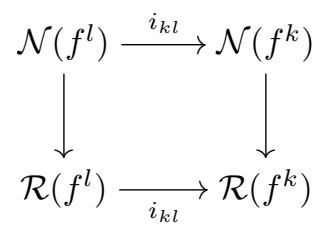

commutes.

The group $\mathbb{Z}_{k}$ acts on $\operatorname{Fix}\left(f^{k}\right)$ by

$$
\operatorname{Fix}\left(f^{k}\right) \ni[x] \rightarrow[f x] \in \operatorname{Fix}\left(f^{k}\right)
$$

and on $\mathcal{R}\left(f^{k}\right)$ by

$$
\mathcal{R}\left(f^{k}\right) \ni[\alpha]^{k} \rightarrow\left[f_{\#}(\alpha)\right]^{k} \in \mathcal{R}\left(f^{k}\right)
$$

Then the diagram

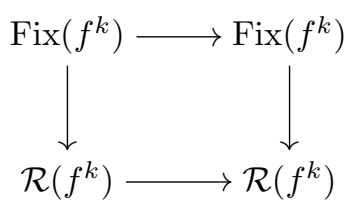

commutes. We denote by $\mathcal{O} \mathcal{R}\left(f^{k}\right)$ the set of orbits of the above action (orbits of Reidemeister classes).

Consider Reidemeister classes $[\alpha]^{k} \in \mathcal{R}\left(f^{k}\right),[\beta]^{l} \in \mathcal{R}\left(f^{l}\right), l \mid k$, satisfying $i_{k l}\left([\beta]^{l}\right)=[\alpha]^{k}$. Then we say that $[\alpha]^{k}$ reduces to $[\beta]^{l}$ or $[\beta]^{l}$ precedes $[\alpha]^{k}$ and we write $[\beta]^{l} \preceq[\alpha]^{k}$. The class is called reducible if $[\beta]^{l} \preceq[\alpha]^{k}$ for a $[\beta]^{l} \in \mathcal{R}\left(f^{l}\right)$, $l<k$. A similar definition works for orbits.

Let $\mathcal{E R}\left(f^{k}\right), \mathcal{I} \mathcal{R}\left(f^{k}\right)$ denote sets of essential and irreducible classes, respectively.

A map $f$ is called essentially reducible if each class $[\alpha]^{l} \in \mathcal{R}\left(f^{l}\right)$ preceding an essential class is essential.

We will denote by $\mathcal{I E O} \mathcal{R}(f)$, or simply $\mathcal{I E O R}$, the set of irreducible essential orbits of Reidemeister classes of $f$.

We define the depth of a Nielsen class $[\alpha]^{k} \in \mathcal{R}\left(f^{k}\right)$ as the least $l \in \mathbb{N}$ so that $[\alpha]^{k}$ reduces to an element in $\mathcal{R}\left(f^{l}\right)$. We will use the same definition for orbits of Reidemeister classes. 
2.1. Nielsen theory for self-maps of tori. Since $T^{m}$ is a $K\left(\mathbb{Z}^{m}, 1\right)$ space, there is a natural bijection

$$
\left[T^{m}, T^{m}\right] \ni[f] \rightarrow f_{\#} \in \operatorname{Hom}_{\mathbb{Z}}\left(\pi_{1} T^{m} ; \pi_{1} T^{m}\right)
$$

where $\left[T^{m}, T^{m}\right]$ denotes the set of homotopy classes and $f_{\#}$ is the fundamental group homomorphism of $\pi_{1} T^{m}=\mathbb{Z}^{m}$. Let $A_{f}$ denote $m \times m$-matrix representing this homomorphism.

Since $\pi_{1} T^{m}=\mathbb{Z}^{m}$ is abelian, $\mathcal{R}(f)=\mathbb{Z}^{m} / \mathrm{im}\left(I-f_{\#}\right)$ is a group. The maps (homomorphisms) $i_{k l}: \mathcal{R}\left(f^{l}\right) \rightarrow \mathcal{R}\left(f^{k}\right)$ are given by

$$
i_{k l}[v]^{l}=\left[\left(I+A_{f}^{l}+\ldots+A_{f}^{k-l}\right) v\right]^{k} .
$$

By [2] $L(f)=\operatorname{det}\left(I-A_{f}\right)$ and $N(f)=|L(f)|$ for each self map of a torus. On the other hand tori are Jiang spaces i.e. all Reidemeister classes of $f: T^{m} \rightarrow T^{m}$ have the same index. By the above, in the case of a self-map $f: T^{m} \rightarrow T^{m}$ of a torus this common index is equal to the sign of the determinant: $\operatorname{sign}\left(\operatorname{det}\left(I-A_{f}\right)\right)$.

We will also base on the following properties of self-maps of a torus $f: T^{m} \rightarrow$ $T^{m}$ (see [11], [12], [17]):

(1) each map $f: T^{m} \rightarrow T^{m}$ is essentially reducible,

(2) for a fixed $k \in \mathbb{N}$ the length and the depth of all essential orbits in $\mathcal{O} \mathcal{R}\left(f^{k}\right)$ are equal,

(3) if $i_{n k}[\alpha]^{k}=i_{n k}[\beta]^{k}$ is essential then $[\alpha]^{k}=[\beta]^{k}$,

(4) each $f$ is essentially reducible to the greatest common divisors (GCD for a shorthand): i.e. $f$ is essentially reducible and whenever an essential class $[\alpha]^{a}$ reduces to both $[\beta]^{b}$ and $[\gamma]^{c}$ then there exists a $[\delta]^{d} \in \mathcal{R}\left(f^{d}\right)$, where $d=\operatorname{gcd}(b, c)$, to which both $[\beta]^{b}$ and $[\gamma]^{c}$ reduce,

(5) essential reducibility to the GCD implies that each essential class $[\alpha]^{a}$ reduces to the unique essential irreducible class $[\beta]^{b} \in \mathcal{I E R}\left(f^{k}\right)$ where $b=\operatorname{depth}\left([\alpha]^{a}\right)$.

2.2. Sequences of Lefschetz numbers. Let $f: T^{m} \rightarrow T^{m}$ be a self-map. Then, by [2], $L\left(f^{k}\right)=\operatorname{det}\left(I-A_{f}^{k}\right)=\left(1-\lambda_{1}^{k}\right) \ldots\left(1-\lambda_{1}^{m}\right)$ where $\lambda_{1}, \ldots, \lambda_{m}$ are eigen-values of $A_{f}$. As we have noticed the index of each Reidemeister class $[\alpha]^{k} \in \mathcal{R}\left(f^{k}\right)$ equals ind $\left(f^{k} ;[\alpha]^{k}\right)=\operatorname{sign}\left(I-A_{f}^{k}\right)$. Thus index of each orbit $[\alpha]^{k} \in \mathcal{O} \mathcal{R}\left(f^{k}\right)$ equals ind $\left(f^{k} ;[\alpha]^{k}\right)=\operatorname{sign}\left(I-A_{f}^{k}\right) \cdot \operatorname{depth}\left([\alpha]^{k}\right)$.

LEMma 2.1. Let $f$ be a self-map of $m$-torus. Let $d_{1}, \ldots, d_{s}$ be the minimal periods of roots of unity of elements in $\operatorname{Spec}\left(A_{f}\right)$. Then the common value of indices of all Reidemeister classes in $\mathcal{R}\left(f^{k}\right)$ equals

$$
r_{k}= \begin{cases}0 & \text { if } d_{i} \mid k \text { for an } i=1, \ldots, s, \\ (-1)^{\gamma_{+}} & \text {if } d_{i} \mid k \text { for no } i=1, \ldots, s \text { and } k \text { is odd, } \\ (-1)^{\gamma_{+}+\gamma_{-}} & \text {if } d_{i} \mid k \text { for no } i=1, \ldots, s \text { and } k \text { is even, }\end{cases}
$$


here $\gamma_{+}\left(\gamma_{-}\right)$denote the numbers of real eigen-values $>+1(<-1)$, respectively.

In other words $r_{k}=0$, if a minimal period of roots of unity in the spectrum divides $n$ and for the remaining $n$ the sequence is the restriction of a sequence from: $a_{k} \equiv+1, b_{k} \equiv-1, c_{k}=(-1)^{k+1}$ or $d_{k}=(-1)^{k}$.

\section{Indices of iterations of a smooth map}

In 1983 Albrecht Dold [5] noticed that the sequence of fixed point indices $A_{k}=\operatorname{ind}\left(f^{k} ; x_{0}\right)$, where $f$ is a continuous self-map of a Euclidean space $\mathbb{R}^{m}$ and $x_{0}$ is an isolated fixed point for each $k$, must satisfy some congruences. Namely, for each $n \in \mathbb{N}$,

$$
\sum_{k \mid n} \mu(n / k) \cdot \operatorname{ind}\left(f^{k} ; x_{0}\right) \equiv 0 \quad(\text { modulo } n)
$$

where $\mu$ denotes the Möbius function.

It was shown [1] that each sequence of integers $\left(A_{k}\right)$ satisfying Dold congruences can be realized as $A_{k}=\operatorname{ind}\left(f^{k} ; x_{0}\right)$, for a continuous self-map of $\mathbb{R}^{m}$ for $m \geq 3$. In other words Dold congruences are the only restrictions for the sequence of the fixed point index of a continuous map.

Surprisingly it turned out that there are much more restrictions on the sequences $A_{k}=\operatorname{ind}\left(f^{k} ; x_{0}\right)$ when $f$ is smooth [19], [4]. In Theorem 3.3 we enlist all possible sequences which can be obtained as fixed point indices of a smooth self-map of $\mathbb{R}^{m}$ (for $m \geq 3$ ).

Definition 3.1. A sequence of integers which occurs in the list in Theorem 3.3 will be called smoothly realizable in $\mathbb{R}^{m}$ (or in dimension $m$ ).

It is convenient to present the sequences of integers as the sum of the following elementary periodic sequences

Definition 3.2. For a given $k \in \mathbb{N}$ we define

$$
\operatorname{reg}_{k}(n)= \begin{cases}k & \text { if } k \mid n, \\ 0 & \text { if } k \nmid n .\end{cases}
$$

In other words, reg $_{k}$ is the periodic sequence:

$$
(0, \ldots, 0, k, 0, \ldots, 0, k, \ldots),
$$

where the non-zero entries appear for indices divisible by $k$.

It turns out that each integer sequence $\left(A_{n}\right)$ can be written down uniquely in the following form of a periodic expansion:

$$
A_{n}=\sum_{k=1}^{\infty} a_{k} \operatorname{reg}_{k}(n), \quad \text { where } a_{n}=\frac{1}{n} \sum_{k \mid n} \mu\left(\frac{n}{k}\right) \operatorname{ind}\left(f^{k}, 0\right) .
$$


Moreover, all coefficients $a_{k}$ are integers if and only if the sequence $\left(A_{n}\right)$ satisfies Dold congruences.

For a finite subset $A \subset \mathbb{Z}$ we denote by $\operatorname{lcm}(A)$ the least common multiplicity of its elements and we denote $\operatorname{LCM}(A)=\{\operatorname{lcm}(B) ; B \subset A\}$. We denote by $L(s)=\operatorname{LCM}(L)$ where $L \subset\{3,4, \ldots\}$ is of cardinality $s$. Moreover, we denote $L_{2}(s)=\operatorname{LCM}(L \cup\{2\})$.

Theorem 3.3 (Main Theorem I in [8]). Let $U \subset \mathbb{R}^{m}$, where $m \geq 3$, be an open neighborhood of 0 and $f: U \rightarrow \mathbb{R}^{m}$ be a $C^{1}$ map having 0 as an isolated fixed point for each iteration. Then the sequence of local indices of iterations $\left\{\operatorname{ind}\left(f^{n}, 0\right)\right\}_{n=1}^{\infty}$ has one of the following forms:

(a) For $m$ odd:

$$
\operatorname{ind}\left(f^{n}, 0\right)=\sum_{k \in L_{2}((m-3) / 2)} a_{k} \operatorname{reg}_{k}(n) .
$$

$\left(\mathrm{B}^{\circ}\right),\left(\mathrm{C}^{\circ}\right),\left(\mathrm{D}^{\circ}\right) \quad \operatorname{ind}\left(f^{n}, 0\right)=\sum_{k \in L((m-1) / 2)} a_{k} \mathrm{reg}_{k}(n)$,

where

$$
a_{1}= \begin{cases}1 & \text { in the case }\left(\mathrm{B}^{\circ}\right), \\ -1 & \text { in the case }\left(\mathrm{C}^{\circ}\right) \\ 0 & \text { in the case }\left(\mathrm{D}^{\circ}\right) .\end{cases}
$$

$\left(\mathrm{E}^{\mathrm{o}}\right),\left(\mathrm{F}^{\mathrm{o}}\right)$

$$
\operatorname{ind}\left(f^{n}, 0\right)=\sum_{k \in L_{2}((m-1) / 2)} a_{k} \operatorname{reg}_{k}(n),
$$

where $a_{1}=1$ and

$$
a_{2}= \begin{cases}0 & \text { in the case }\left(\mathrm{E}^{\mathrm{O}}\right), \\ -1 \quad \text { in the case }\left(\mathrm{F}^{\mathrm{O}}\right) .\end{cases}
$$

(b) For $m$ even:

$$
\begin{aligned}
& \operatorname{ind}\left(f^{n}, 0\right)=\sum_{k \in L_{2}((m-4) / 2)} a_{k} \operatorname{reg}_{k}(n) . \\
& \operatorname{ind}\left(f^{n}, 0\right)=\sum_{k \in L((m-2) / 2)} a_{k} \operatorname{reg}_{k}(n) . \\
& \operatorname{ind}\left(f^{n}, 0\right)=\sum_{k \in L_{2}((m-2) / 2)} a_{k} \operatorname{reg}_{k}(n),
\end{aligned}
$$

$\left(\mathrm{C}^{\mathrm{e}}\right),\left(\mathrm{D}^{\mathrm{e}}\right),\left(\mathrm{E}^{\mathrm{e}}\right)$

where

$$
a_{1}= \begin{cases}1 & \text { in the case }\left(\mathrm{C}^{\mathrm{e}}\right) \\ -1 & \text { in the case }\left(\mathrm{D}^{\mathrm{e}}\right) \\ 0 & \text { in the case }\left(\mathrm{E}^{\mathrm{e}}\right)\end{cases}
$$




$$
\operatorname{ind}\left(f^{n}, 0\right)=\sum_{k \in L(m / 2)} a_{k} \operatorname{reg}_{k}(n)
$$

where $a_{1}=1$.

REMARK 3.4. Later we will be also interested in iterations $f^{k}$, for $k$ dividing a prescribed $n$, so will consider only finite sequences labeled by $\{k \in \mathbb{N} ; k \mid n\}$. We will say that a finite sequence $\left(B_{k}\right)_{k \mid n}$ is smoothly realizable in $\mathbb{R}^{m}$ if it is a restriction of a sequence in the list in Theorem 3.3.

\section{Condition for the realizability of the minimal number of periodic points by a smooth map}

Let $f: M \rightarrow M$ be a continuous self-map of a smooth manifold and let $n \in \mathbb{N}$. Let

$$
M F_{n}(f)=\min _{g \sim f} \operatorname{Fix}\left(g^{n}\right)
$$

be the minimal number of $n$-periodic points where $g$ runs through the set of all continuous maps homotopic to $f$. An algebraically computable lower bound $N F_{n}(f) \leq M F_{n}(f)$ was introduced by Boju Jiang [17]. Later it turned out that the equality $N F_{n}(f)=M F_{n}(f)$ holds for all self-maps of compact manifolds of dimension $\geq 3$ [13]. On the other hand a theory investigating the least number of $n$-periodic in the smooth homotopy class $N J D_{n}(f)$ appeared in [6], [7]. It turned out that in general, the gap between the two numbers may be very large [6].

In this paper we confine to essentially reducible maps $f: X \rightarrow X$. Then each essential irreducible orbit in $\mathcal{I E O R}\left(g^{k}\right)$ of any map $g$ homotopic to $f$, must contain at least $\mathcal{I E O R}\left(f^{k}\right) \cdot k$ periodic points and moreover these orbits are mutually disjoint. Thus

Lemma 4.1 (comp. Corollary (7.2) in [15]). Let $f: X \rightarrow X$ be an essentially reducible map of a finite polyhedron. Then

$$
\# \operatorname{Fix}\left(g^{n}\right) \geq \sum_{k \mid n} \# \mathcal{I} \mathcal{E O} \mathcal{R}\left(f^{k}\right) \cdot k
$$

so the sum $\sum_{k \mid n} \# \mathcal{I E O R}\left(f^{k}\right) \cdot k$ is a lower bound of the number of $n$-periodic points in the homotopy class of $f$. Moreover, the equality holds if and only if each orbit of points in $\operatorname{Fix}\left(f^{k}\right)$ represents an orbit in $\mathcal{I E O R}$ and no other point represents this orbit.

Moreover, for essentially reducible maps the equality

$$
N F_{n}(f)=\sum_{k \mid n} \# \mathcal{I E} \mathcal{O} \mathcal{R}\left(f^{k}\right) \cdot k
$$


holds so, by [13], in dimension $\geq 3$ the equality

$$
\# \operatorname{Fix}\left(g^{n}\right)=\sum_{k \mid n} \# \mathcal{I} \mathcal{E O} \mathcal{R}\left(f^{k}\right) \cdot k
$$

can be realized by a continuous map $g$ homotopic to $f$. Thus the desired equality $N F_{n}(f)=M F_{n}(f)$ takes place if and only if $f$ is homotopic to a smooth map $g$ realizing the equality (4.1). This implies

Lemma 4.2. Let $f: M \rightarrow M$ be an essentially reducible self-map of a compact manifold of dimension $\geq 3$ and let $n$ be a natural number. Then the least numbers of n-periodic points in the continuous homotopy class and smooth homotopy class of $f$ are equal $\left(N J D_{n}(f)=N F_{n}(f)\right)$ if and only if there exists a smooth map $g$ homotopic to $f$ satisfying

$$
\# \operatorname{Fix}\left(g^{n}\right)=\sum_{k \mid n} \# \mathcal{I} \mathcal{E O} \mathcal{R}\left(f^{k}\right) \cdot k .
$$

\section{Reidemeister graph}

We fix a map $f: M \rightarrow M$. We put all Reidemeister data into a directed graph $\Gamma$.

- Vertices are elements of the disjoint sum $\operatorname{Vert}(\Gamma)=\bigcup_{k \in \mathbb{N}} \mathcal{O R}\left(f^{k}\right)$.

- There is a unique directed edge from $[\alpha]^{a} \in \mathcal{O R}\left(f^{a}\right)$ to $[\beta]^{b} \in \mathcal{O} \mathcal{R}\left(f^{b}\right)$ if $i_{b a}\left([\alpha]^{a}\right)=[\beta]^{b}$.

- Moreover, to each vertex (an orbit of Reidemeister classes) $[\alpha]^{a} \in \mathcal{O} \mathcal{R}\left(f^{a}\right)$ an integer $\operatorname{ind}\left(f^{a} ;[\alpha]^{a}\right)$ is defined, and the following Dold Congruences are satisfied (Lemma 3.3 in $[7]$ ): for each $[\beta]^{b} \in \mathcal{O} \mathcal{R}\left(f^{b}\right)$ :

$$
\left.\sum_{[\gamma]^{c} \preceq[\beta]^{b}} \mu(b / c) \cdot \operatorname{ind}\left(f^{c}:[\gamma]^{c}\right) \equiv 0 \quad \text { (modulo } b\right)
$$

where $\mu$ denotes the Mobius function and $[\gamma]^{c} \in \mathcal{O} \mathcal{R}\left(f^{c}\right)$ runs over the family of all Reidemeister classes preceding $[\beta]^{b}$.

REMARK 5.1. Let us notice that if $M$ is simply-connected then for each multiplicity of $f$ there is only one Reidemeister class, hence $\operatorname{Vert}(f)=\mathbb{N}$. Now the function

$$
\operatorname{Vert}(f)=\bigcup_{k \in \mathbb{N}} \mathcal{O R}\left(f^{k}\right) \supset \mathcal{O} \mathcal{R}\left(f^{k}\right) \ni[\gamma]^{k} \rightarrow \operatorname{ind}\left(f^{k} ;[\gamma]^{k}\right) \in \mathbb{Z}
$$

reduces to the sequence $\operatorname{ind}\left(f^{k}\right)=L\left(f^{k}\right)$.

Now we consider a smooth map $f: M \rightarrow M$ and its isolated orbit $\alpha=$ $\left\{x_{1}, \ldots, x_{a}\right\}$ consisting of $a$ points. They give rise to

(1) an orbit of Reidemeister classes $[\alpha]^{a} \in \mathcal{O} \mathcal{R}\left(f^{a}\right)$, 
(2) a function $C_{\alpha}$ : $\operatorname{Vert}(f) \rightarrow \mathbb{Z}$ given by

$$
\mathcal{O R}\left(f^{b}\right) \ni[\beta]^{b} \rightarrow \operatorname{ind}\left(f^{b} ; \alpha \cap[\beta]^{b}\right) \in \mathbb{Z} .
$$

Then, by the commutativity of the fixed point index,

$$
C_{\alpha}\left([\beta]^{b}\right)= \begin{cases}a \cdot \operatorname{ind}\left(f^{b} ; x_{1}\right) & \text { for }[\alpha]^{a} \preceq[\beta]^{b}, \\ 0 & \text { otherwise }\end{cases}
$$

where $[\beta]^{b} \in \mathcal{O} \mathcal{R}\left(f^{b}\right)$.

On the other hand, for an $[\alpha]^{a} \in \mathcal{O} \mathcal{R}\left(f^{a}\right)$ and a number $r \in \mathbb{N}$, we define $\operatorname{Reg}_{[\alpha]^{a}}^{r}: \operatorname{Vert}\left(f^{a}\right) \rightarrow \mathbb{Z}$ by the formula

$$
\operatorname{Reg}_{[\alpha]^{a}}^{r}\left([\beta]^{b}\right)= \begin{cases}a \cdot r & \text { if } i_{r a, a}\left([\alpha]^{a}\right) \preceq[\beta]^{b}, \\ 0 & \text { otherwise. }\end{cases}
$$

More generally, for each Dold sequence $d_{k}=\sum_{l} a_{l} \operatorname{reg}_{l}(k)$ and an orbit $[\alpha]^{a} \in$ $\mathcal{O R}\left(f^{a}\right)$ we define a function $D: \operatorname{Vert}\left(f^{k}\right) \rightarrow \mathbb{Z}$ as $D\left([\beta]^{b}\right)=\sum_{l} a_{l} \operatorname{Reg}_{[\alpha]}^{l}\left([\beta]^{b}\right)$. We say then that the sequence $\sum a_{l} \operatorname{reg}_{l}$ is attached to the orbit $[\alpha]^{a}$ or that $D: \operatorname{Vert}\left(f^{k}\right) \rightarrow \mathbb{Z}$ comes from $\sum a_{l} \mathrm{reg}_{l}$.

Let us notice that the function $C_{a}$ defined above comes from the sequence $a_{l}=\operatorname{ind}\left(f^{l} ; x_{1}\right)$ which is smoothly realizable in $\mathbb{R}^{m}$.

Now we assume that $\operatorname{Fix}\left(f^{n}\right)$ is finite. Then $\operatorname{Fix}\left(f^{n}\right)=\bigcup a$ splits into mutually disjoint orbits of points and of course \# $\operatorname{Fix}\left(f^{n}\right)=\sum_{a} \operatorname{length}(a)$. On the other hand we notice that

$$
\operatorname{ind}\left(f^{k} ;[\beta]^{b}\right)=\sum_{a} \operatorname{ind}\left(f^{k} ; a\right)
$$

where the summation runs through the set of all orbits of points contained in $[\beta]^{b}$. Let us emphasize that each summand $\operatorname{ind}\left(f^{k} ; a\right)$ is coming from a sequence smoothly realizable in $\mathbb{R}^{m}$. Thus the function

$$
\operatorname{Vert}(\Gamma)=\bigcup_{k \in \mathbb{N}} \mathcal{O R}\left(f^{k}\right) \supset \mathcal{O R}\left(f^{k}\right) \ni[\gamma]^{c} \rightarrow \operatorname{ind}\left(f^{k} ;[\gamma]^{c}\right) \in \mathbb{Z}
$$

is the sum of expressions smoothly realizable in $\mathbb{R}^{m}$ attached to vertices (orbits of Reidemeister classes) of $\operatorname{Vert}(\Gamma)$. It turns out that, under the assumption $\operatorname{dim} M \geq 3$, the existence of a special such decomposition is also a sufficient condition for the existence of a smooth map $g$ realizing the minimal number of periodic points.

Theorem 5.2 ([15, Theorem 7.3]). An essentially reducible map $f: M \rightarrow M$ is homotopic to a smooth map $g$ realizing the least number of n-periodic points 
if and only if one can attach to each orbit $[\alpha]^{a} \in \mathcal{I} \mathcal{E O} \mathcal{R}\left(f^{k}\right)(k \mid n)$ a sequence $C_{[\alpha]^{a}}$, realizable in $\mathbb{R}^{m}$, so that

$$
\operatorname{ind}\left(f^{k} ;[\beta]^{b}\right)=\sum_{[\alpha]^{a} \in \mathcal{I} \mathcal{E} \mathcal{O R}} C_{[\alpha]^{a}}\left([\beta]^{b}\right)
$$

for each $[\beta]^{b} \in \mathcal{O} \mathcal{R}\left(f^{k}\right), k \mid n$.

\section{Proof of the Theorem of You}

Now we are in a position to give an algebraic proof of the Theorem of Cheng Ye You.

Theorem $6.1([20])$. Let $m, r \in \mathbb{N}$ and let $f: T^{m} \rightarrow T^{m}$ be a self-map of the $m$-torus. Then the least number of r-periodic points in the homotopy class of $f$

$$
\min \left\{\# \operatorname{Fix}\left(g^{r}\right) ; g \text { is homotopic to } f\right\}
$$

can be realized by a smooth map.

Proof. We will give the algebraic proof in dimension $\geq 3$. The low dimensions will be discussed in the last section. First we consider two trivial cases:

Case 1. There are no roots of unity in spectrum.

Let $\alpha_{k}=\operatorname{sign}\left(\operatorname{det}\left(L\left(f^{k}\right)\right)\right)$. By Lemma 2.1 this sequence is of the form: $1,-1,(-1)^{k}$ or $(-1)^{k+1}$, hence is smoothly realizable in dimension 2 . When there are no roots of unity in spectrum, the Reidemeister graph splits into connected components each isomorphic to $\mathbb{N}$. By the above, each component can be smoothly realized by attaching an expression $\alpha_{k}$ to the least vertex of the component.

Case 2. 1 is an eigenvalue.

Let $f: \mathbb{R}^{m} \rightarrow \mathbb{R}^{m}$ be the linear map representing the given map. Now $V_{0}=\left\{v \in \mathbb{R}^{m} ; f(v)=v\right\}$ is a nonzero subspace. A shift of a small nonzero vector $v_{0} \in V_{0}$ induces a smooth map of $T$ without fixed points.

Thus we may assume that

(1) 1 does not belong to $\operatorname{Spec}\left(A_{f}\right)$,

(2) $\left\{d_{1}, \ldots, d_{s}\right\}, s \geq 1$, denote all primitive degrees of roots of unity in the Spectrum.

First we consider the case when the index of each essential class equals +1 i.e. $\operatorname{ind}\left(f^{b} ;[\beta]^{b}\right)=1$ for each essential class $[\beta]^{b}$. The remaining cases are simple modifications and will be discussed later.

We will give the formula of the decomposition. First a few words about its origin. Let us start with the decomposition as in the case without roots of unity

$$
\operatorname{ind}\left(f^{b} ;[\beta]^{b}\right)=\sum_{A \in \mathcal{I E O} \mathcal{R}} C_{A}\left([\beta]^{b}\right)
$$


where $C_{A}=\operatorname{Reg}_{A}^{1}$, i.e. to each orbit in $\mathcal{I E O R}$ the constant sequence reg ${ }_{1}$ is attached. Then equality (6.1) is satisfied for all essential orbits. However, for some $[\delta]^{d_{i}} \in \mathcal{O} \mathcal{R}\left(f^{d_{i}}\right)$, there may be many $A \in \mathcal{I E O} \mathcal{R}$ preceding $[\delta]^{d_{i}}$ which makes

$$
\sum_{A \in \mathcal{I E O} \mathcal{O}} C_{A}\left([\delta]^{d_{i}}\right)=\sum_{A \in \mathcal{I E O} \mathcal{R}} \operatorname{Reg}_{A}^{1}\left([\delta]^{d_{i}}\right)=\sum_{A \in \mathcal{I E \mathcal { O } R}, A \preceq[\delta]^{d_{i}}} \operatorname{depth}(A)>0
$$

although $\operatorname{ind}\left(f^{d_{i}} ;[\delta]^{d_{i}}\right)=0$.

To get the equality in this case we fix for each $[\delta]^{d_{i}}$ an orbit $A \in \mathcal{I E O R}$ preceding $[\delta]^{d_{i}}$ and we add to $C_{A}$ a suitable multiplicity of $\operatorname{Reg}_{A}^{d_{i} / a_{d_{i}}}$. But this does not guarantee the equality for $[\delta]^{\operatorname{lcm}\left(d_{i_{1}}, d_{i_{2}}\right)} \in \mathcal{O} \mathcal{R}\left(f^{\operatorname{lcm}\left(d_{i_{1}}, d_{i_{2}}\right)}\right)$ and we have to make next corrections following Inclusion-Exclusion arguments. Since the fixed point index here is constant, after $s$ steps (where $s$ is the number of minimal degrees of roots of unity) we get the equality (6.1) for all orbits.

Now we start the formal proof. We define a sum

$$
\begin{aligned}
& S=\sum_{A \in \mathcal{I E O} \mathcal{R}} \operatorname{Reg}_{A}^{1} \\
& +(-1)^{1} \sum_{1 \leq i_{1} \leq s} \sum_{[\delta]^{i_{1}} \in \mathcal{O} \mathcal{R}\left(f^{d_{i_{1}}}\right)}\left(\frac{1}{d_{i_{1}}} \sum_{A \in \mathcal{I} \mathcal{O} \mathcal{O}, A \prec[\delta]^{i_{1}}} \operatorname{depth}(A)\right) \operatorname{Reg}_{i_{d_{i_{1}}, a} A}^{1}
\end{aligned}
$$

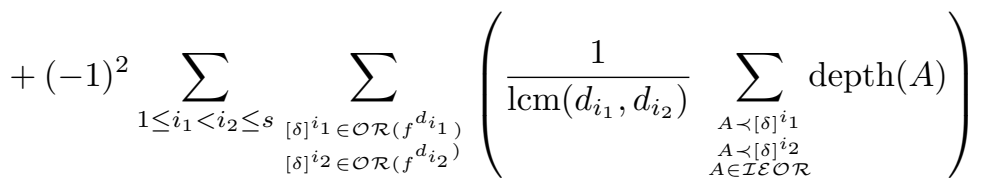

$$
\begin{aligned}
& \times \operatorname{Reg}_{i_{\operatorname{lcm}\left(d_{i_{1}}, d_{i_{2}}\right), a}(A)}^{1}+\ldots \\
& +(-1)^{k} \sum_{\substack{1 \leq i_{1}<\ldots<i_{k} \leq s \\
[\delta]^{i} h \in \mathcal{O} \mathcal{R}\left(f^{d_{i}}\right)}}\left(\frac{1}{\operatorname{lcm}\left(d_{i_{1}}, \ldots, d_{i_{k}}\right)} \sum_{\substack{A<\delta \delta]^{i} h \\
A \in \mathcal{I} \mathcal{O} \mathcal{R}}} \operatorname{depth}(A)\right) \\
& \times \operatorname{Reg}_{i_{\operatorname{lcm}\left(d_{i_{1}}, \ldots, d_{i_{k}}\right), a}(A)}^{1}+\ldots \\
& +(-1)^{s}\left(\frac{1}{\operatorname{lcm}\left(d_{1}, \ldots, d_{s}\right)} \sum_{\substack{A<\left[\delta \delta^{h} h \\
A \in \mathcal{I} \mathcal{E} \mathcal{R}\right.}} \operatorname{depth}(A)\right) \operatorname{Reg}_{i_{\operatorname{lcm}^{\prime}\left(d_{1}, \ldots, d_{s}\right), a}(A) .}^{1} .
\end{aligned}
$$

Here $A \in \mathcal{I E O R}\left(f^{a}\right)$ denotes an arbitrarily chosen orbit preceding the orbits $[\delta]^{i_{1}}, \ldots,[\delta]^{i_{k}}$, i.e. an orbit in the summation set of the corresponding sum. Then $i_{\operatorname{lcm}\left(d_{i_{1}}, \ldots, d_{i_{k}}\right), a}(A)$ is the least orbit reducing to all $[\delta]^{i_{1}}, \ldots,[\delta]^{i_{k}}$. (One might call $i_{\operatorname{lcm}\left(d_{i_{1}}, \ldots, d_{i_{k}}\right), a}(A)$ the least common multiplicity of $[\delta]^{i_{1}}, \ldots,[\delta]^{i_{k}}$ with the respect to $\preceq$ relation.) It is easy to check that $i_{\operatorname{lcm}\left(d_{i_{1}}, \ldots, d_{i_{k}}\right), a}(A)$ does not depend on the choice of $A$. We will show that

(1) $S\left([\beta]^{b}\right)=\operatorname{ind}\left(f^{b} ; B\right)$ for each $[\beta]^{b} \in \mathcal{O} \mathcal{R}\left(f^{b}\right)$. 
(2) $S$ splits to the sum of expressions smoothly realizable in $\mathbb{R}^{m}$ attached at different elements of $\mathcal{I E O R}$.

Then the assumptions of Theorem 5.2 are satisfied so the index function is smoothly realizable in $\mathbb{R}^{m}$ as required. This will end the proof of the Theorem for $\operatorname{ind}\left(f^{b} ;[\beta]^{b}\right)=1$.

We emphasize that we do not claim that all coefficients in the above sum are integers. However, after regrouping the coefficients standing at the same Regs, the above equality (1) and the uniqueness of the Dold expansion give the sum as above with integer coefficients.

(1) If $[\beta]^{b} \in \mathcal{O} \mathcal{R}\left(f^{b}\right)$ is essential then it reduces to single class $\left[\alpha_{0}\right] \in \mathcal{I E} \mathcal{O} \mathcal{R}$, hence

$$
S\left([\beta]^{b}\right)=\operatorname{Reg}_{\left[\alpha_{0}\right]}^{1}\left([\beta]^{b}\right)=\operatorname{depth}\left(\left[\alpha_{0}\right]\right)=\operatorname{ind}\left(f^{b} ;[\beta]^{b}\right) .
$$

If $[\beta]^{b}$ is inessential and does not reduce to any essential orbit then both sides are 0 .

Let $[\beta]^{b} \in \mathcal{O} \mathcal{R}\left(f^{b}\right)$ be inessential and reduce to an essential class. It remains to show that $S\left([\beta]^{b}\right)=0$. Without loss of generality we may assume that $[\delta]^{i_{h}} \preceq$ $[\beta]^{b}$ if and only if $i_{h} \leq s_{0}$ (for an $s_{0} \leq s$ ). Now $S\left([\beta]^{b}\right)$ expands to sum (6.2) with $s=s_{0}$.

Let us emphasize that in each inner sum the summation runs over a subset of $\mathcal{I E O R}$. We will show that the contribution in $S\left([\beta]^{b}\right)$ of the summands reducing to the same $[\alpha] \in \mathcal{I E O} \mathcal{R}$ is zero. Let us fix such $[\alpha] \in \mathcal{I E O} \mathcal{R},[\alpha] \in \mathcal{O} \mathcal{R}\left(f^{a}\right)$. We may assume that $a \mid d_{i}$ if and only if $i \leq s_{1}$ for an $s_{1} \leq s_{0}$. Now the $k$-th sum reduces to the same sum as above: we only replace $s_{0}$ by $s_{1}$

$$
\begin{aligned}
(-1)^{k} \sum_{\substack{1 \leq i_{1}<\ldots<i_{k} \leq s_{1} \\
[\delta]^{i} h \in \mathcal{O} \mathcal{R}\left(f^{d_{i}}\right)}}\left(\frac{1}{\operatorname{lcm}\left(d_{i_{1}}, \ldots, d_{i_{k}}\right)} \sum_{\substack{A \prec[\delta]^{i} h \\
A \in \mathcal{I} \mathcal{E} \mathcal{R}}} \operatorname{depth}(A)\right) \\
\times \operatorname{Reg}_{i_{i_{\mathrm{cm}}\left(d_{i_{1}}, \ldots, d_{i_{k}}\right), a}}^{1}(A) .
\end{aligned}
$$

In particular if with take the sum over the fixed $[\alpha] \in \mathcal{I E} \mathcal{O} \mathcal{R}$ then we get

$$
(-1)^{k} \sum_{\substack{[\delta]^{i} h \in \mathcal{O R}\left(f^{d_{i_{h}}}\right) \\
1 \leq d_{i_{1}}<\ldots<d_{i_{k}} \leq s_{1}}} \operatorname{depth}([\alpha])=(-1)^{k}\left(\begin{array}{c}
s_{1} \\
k
\end{array}\right) \cdot \operatorname{depth}([\alpha]) .
$$

Thus the contribution of all summands over $[\alpha]$ in the total sum equals

$$
\begin{aligned}
\operatorname{depth}([\alpha]) & +(-1)^{1}\left(\begin{array}{c}
s_{1} \\
1
\end{array}\right) \operatorname{depth}([\alpha]) \\
& +(-1)^{2}\left(\begin{array}{c}
s_{1} \\
2
\end{array}\right) \operatorname{depth}([\alpha])+\ldots+(-1)^{s_{1}}\left(\begin{array}{c}
s_{1} \\
s_{1}
\end{array}\right) \operatorname{depth}([\alpha])
\end{aligned}
$$




$$
\begin{aligned}
& =\operatorname{depth}([\alpha])\left(\left(\begin{array}{c}
s_{1} \\
0
\end{array}\right)+(-1)^{1}\left(\begin{array}{c}
s_{1} \\
1
\end{array}\right)+(-1)^{2}\left(\begin{array}{c}
s_{1} \\
2
\end{array}\right)+\ldots+(-1)^{s_{1}}\left(\begin{array}{c}
s_{1} \\
s_{1}
\end{array}\right)\right) \\
& =(1-1)^{s_{1}} \cdot \operatorname{depth}([\alpha])=0 .
\end{aligned}
$$

(2) Now we show that sum (6.3) splits to smoothly realizable expressions. We may rewrite (6.3) as

$$
\begin{aligned}
& S=\sum_{[\alpha] \in \mathcal{I E} \mathcal{O R}} \operatorname{Reg}_{[\alpha]}^{1}+\sum_{[\alpha] \in \mathcal{I E} \mathcal{O} \mathcal{R}} \sum_{1 \leq i_{1} \leq s_{1}}(\ldots) \operatorname{Reg}_{i_{d_{1}}, a}([\alpha]) \\
& +\sum_{[\alpha] \in \mathcal{I E O} \mathcal{O R}} \sum_{1 \leq i_{1} \leq i_{2} \leq s_{1}}(\ldots) \operatorname{Reg}_{i_{\operatorname{lcm}\left(d_{i_{1}}, d_{i_{2}}\right), a}([\alpha])}+\ldots \\
& +\sum_{[\alpha] \in \mathcal{I} \mathcal{E} \mathcal{O R}} \sum_{1 \leq i_{1} \leq \ldots \leq i_{k} \leq s_{1}}(\ldots) \operatorname{Reg}_{i_{\operatorname{lcm}\left(d_{i_{1}}, \ldots, d_{i_{k}}\right), a}([\alpha])}+\ldots \\
& +\sum_{[\alpha] \in \mathcal{I E} \mathcal{O} \mathcal{R}}(\ldots) \operatorname{Reg}_{i_{\operatorname{lcm}\left(d_{1}, \ldots, d_{s_{1}}\right), a}([\alpha])}
\end{aligned}
$$

where $(. .$.$) denote some constants.$

Now the summands may be regrouped to

$$
\begin{aligned}
& \sum_{[\alpha] \in \mathcal{I E O} \mathcal{R}}\left[\operatorname{Reg}_{[\alpha]}^{1}+\sum_{1 \leq i_{1} \leq s_{1}}(\ldots) \operatorname{Reg}_{i_{d_{i_{1}}, a}([\alpha])}\right. \\
& +\sum_{1 \leq i_{1} \leq i_{2} \leq s_{1}}(\ldots) \operatorname{Reg}_{i_{l c m\left(d_{i_{1}}, d_{i_{2}}\right), \alpha}([\alpha])}+\ldots \\
& +\sum_{1 \leq i_{1} \leq \ldots \leq i_{k} \leq s_{1}}(\ldots) \operatorname{Reg}_{i_{\operatorname{lem}\left(d_{i_{1}}, \ldots, d_{i_{k}}\right), \alpha}([\alpha])}+\ldots
\end{aligned}
$$

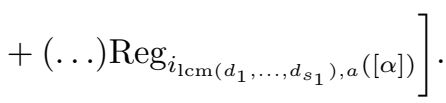

We do not claim that the constants must be integers. However, we may assume that in the above sum the coefficient standing at the same $\operatorname{Reg}_{i_{\operatorname{lcm}\left(d_{i_{1}}, \ldots, d_{i_{k}}\right), a}([\alpha])}$, coming from different $[\alpha]$, are different than zero at most once, since otherwise we may coalesce them. Now the equality $\left.S\left([\beta]^{b}\right)=\operatorname{ind}\left(f^{[} \beta\right]^{b} ;\right)$ and the uniqueness of Dold's expansion give the integer coefficients (...).

Finally, we notice that each inner sum, (i.e. the sum for a fixed $[\alpha]$ ) is smoothly realizable in $\mathbb{R}^{m}$, where $m=2 s$, as $\left(F^{e}\right)$ in Theorem 3.3. Since $2 s$ is less or equal to the dimension of the considered torus, we get the desired splitting into smoothly realizable expressions. This ends the proof for the case $\operatorname{ind}\left(f^{b} ;[\beta]^{b}\right) \geq 0$.

Now we assume that $\operatorname{ind}\left(f^{b} ;[\beta]^{b}\right)=(-1)^{b+1}$. Then there must be at least one real eigen-value $<-1$, which implies $m \geq 2 s+1$.

Now we consider the same sum as above where each $\operatorname{Reg}_{[\gamma]^{c}}^{1}$ is replaced by $\operatorname{Reg}_{[\gamma]^{\mathrm{c}}}^{1}-\operatorname{Reg}_{[\gamma]^{\mathrm{c}}}^{2}$. Then the proof goes exactly as in the previous case. Finally, 
we get the splitting of the sum into

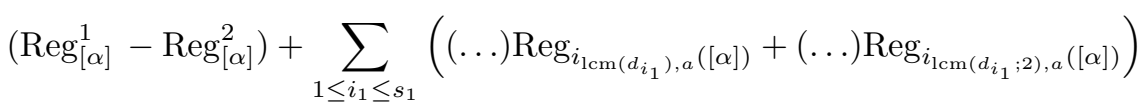

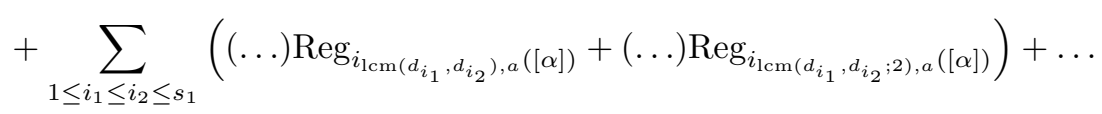

$$
\begin{aligned}
& +\sum_{1 \leq i_{1} \leq \ldots \leq i_{k} \leq s_{1}}\left((\ldots) \operatorname{Reg}_{i_{\operatorname{lcm}\left(d_{i_{1}}, \ldots, d_{i_{k}}\right), a}([\alpha])}\right. \\
& \left.+(\ldots) \operatorname{Reg}_{i_{\operatorname{lcm}\left(d_{i_{1}}, \ldots, d_{i_{k}} ; 2\right), a}([\alpha])}\right)+\ldots \\
& +\left((\ldots) \operatorname{Reg}_{i_{\operatorname{lcm}\left(d_{1}, \ldots, d_{s_{1}}, a\right.}([\alpha])}+(\ldots) \operatorname{Reg}_{\left.i_{\operatorname{lcm}\left(d_{1}, \ldots, d_{\left.s_{1}, 2\right), a}([\alpha])\right.}\right) .} .\right.
\end{aligned}
$$

Now we notice that this is the sum $\left(\mathrm{F}^{\circ}\right)$ or $\left(\mathrm{D}^{\mathrm{e}}\right)$ in Theorem 3.3 .

Let ind $\left(f^{b} ;[\beta]^{b}\right)=-1$. Then $m \geq 2 s+1$. We consider the original sum with the opposite coefficients, we follow the case ind $=+1$ and we get the splitting into sums starting from $-\operatorname{Reg}_{[\alpha]}^{1}+(\ldots)$. These are expressions $\left(\mathrm{C}^{\circ}\right)$ smoothly realizable in dimension $2 s+1 \leq m$.

Let ind $\left(f^{b} ;[\beta]^{b}\right)=(-1)^{b}$. Then $m \geq 2 s+2$. We get the splitting into similar sums starting from $-\operatorname{Reg}_{[\alpha]}^{1}-\operatorname{Reg}_{[\alpha]}^{2}+(\ldots)$. These are expressions $\left(\mathrm{A}^{\circ}\right)$ smoothly realizable in dimension $2 s+2 \leq m$.

\section{Self-maps of low dimensional tori}

Since Theorem 5.2 requires the assumption $m \geq 3$, the cases of lower dimensions need a separate, much simpler, explanation. To get the proof of Theorem 6.1 in all dimensions independent of [20], we will present some well known deformations with the emphasize that all involving homotopies can be smooth. The unique nontrivial argument, due to B. Haplern, is used in the case of complex eigen-values of the linear map inducing $f: T^{2} \rightarrow T^{2}$,

Let $f: T^{1} \rightarrow T^{1}$ be a self-map of degree $d$ and let $k$ be an integer. If $d^{k} \neq 1$ then the $k$-th iteration of the smooth map $f_{d}(z)=z^{d}$ realizes the Nielsen number

$$
N\left(f^{k}\right)=N\left(f_{d}^{k}\right)=N\left(f_{d^{k}}\right)=\left|1-d^{k}\right|
$$

which obviously is lower bound of the number of $k$-periodic points.

Now it remains to consider the cases $d=1$ and $d=-1$. When $d=1$ then $f$ may be taken as the identity on $T^{1}$ and an irrational twist removes all periodic points.

Finally, we consider $f(z)=\bar{z}$, the map of degree -1 . If $f^{\prime}(z)$ denotes a smooth deformation of $f$ such that $\operatorname{re}\left(f^{\prime}(z)\right)>\operatorname{re}(z)$ for $\operatorname{im}(z)>0$ then all iterations of $f^{\prime}$ have exactly two fixed points \pm 1 , hence $f^{\prime}$ is the desired map. 
Now we consider self-maps of $T^{2}$. Each such map is homotopic to a map induced by a linear map $A: \mathbb{R}^{2} \rightarrow \mathbb{R}^{2}$. Now

$$
L(f)=\operatorname{det}(I-A)=\left(1-\lambda_{1}\right)\left(1-\lambda_{2}\right),
$$

where $\lambda_{1}, \lambda_{2}$ are eigen-values of $A$. If $\lambda_{1}^{k} \neq 1$ and $\lambda_{2}^{k} \neq 1$ then the self-map $f_{A}$ of $T^{2}$ induced by the linear (hence smooth) map $A$ satisfies $\# \operatorname{Fix}\left(f_{A}^{k}\right)=N\left(f^{k}\right)$ thus $f_{A}$ realizes the least number of $k$-periodic points.

It remains to consider the cases when $\lambda_{i}$ is a root of unity.

Case $1 .+1$ is an eigen-value.

Then we may assume that $A=\left(\begin{array}{ll}a & 0 \\ b & 1\end{array}\right)$ for some $a, b \in \mathbb{Z}$. The map $\rho(x, y)=x$ defines the fibration $\rho: T^{2} \rightarrow T^{1}$ and $f_{A}$ defines the fibre map over the map $\bar{f}_{A}[x]=[a x]$. We notice that the degree of the restrictions to the fibres is +1 .

We may deform $\bar{f}$ to a map with the finite number of $k$-periodic points and then to remove all $k$-periodic points of $f$, since the degree on the fibre is +1 .

Case 2. -1 is an eigen-value.

Now we may take $A=\left(\begin{array}{cc}a & 0 \\ b & -1\end{array}\right)$. This gives the fibre map over the map $\bar{f}_{A}[x]=[a x]$, as above but now the degree of each fibre map is -1 .

Assume moreover that $a \neq \pm 1$. Let us present $k=2^{s} \cdot p$ where $p$ is odd. Consider the base map $\bar{f}[x]=[a x]$. Now $\operatorname{Fix}\left(\bar{f}^{p}\right)$ contains $\left|a^{p}-1\right|$ points and the remaining points in $\operatorname{Fix}\left(\bar{f}^{k}\right)$ belong to orbits of even length. The degree of the restriction of $f^{l}$ to each fibre has degree $(-1)^{l}$. Thus a smooth deformation of $f$, with the support near the fibres over k-periodic points, reduces the number of periodic points in each fibre, over an orbit of odd length, to 2 and removes all $k$-periodic points in fibres lying over orbits of even length. Thus we get $\# \operatorname{Fix}\left(f_{1}^{k}\right)=2\left|a^{p}-1\right|=\left|\operatorname{det}\left(I-A^{p}\right)\right|=N\left(f_{1}^{p}\right)$. Since $p \mid k, N\left(f_{1}^{p}\right) \leq N F_{n}(f)$ hence the smooth map $f_{1}$ realizes the least number of $n$ periodic points in the homotopy class of $f$.

If $a=1$ then the base map $\bar{f}$ is homotopic to a periodic points free map and the Covering Homotopy Property removes all periodic points of $f$.

If $a=-1$ then $\bar{f}$ is homotopic to a map with exactly two periodic points and the local homotopy allows to minimize the periodic points in the fibres over these two points.

Case 3. $\lambda$ is a complex root of unity.

Let $k$ be the minimal period of $\lambda$. We may assume that $f$ is induced by a linear map also denoted by $f$. Since $\lambda^{k}=1$ and dimension is $2, f^{k}$ is the identity. On the other hand $\operatorname{Fix}\left(f^{l}\right)$ is finite for all $l \mid k, l \neq k$. Thus we may apply Theorem 3 in [10]. There exists a smooth deformation $f_{t}$ starting from $f_{0}=f$ to a map $f_{1}$ such that

$$
\operatorname{Fix}\left(f_{1}^{k}\right)=\bigcup_{l} \operatorname{Fix}\left(f^{l}\right)
$$


where the summation runs over the set $\{l \in \mathbb{N} ; l \mid k, l \neq k\}$.

It remains to show that $\operatorname{Fix}\left(f_{1}^{k}\right)=N F_{k}(f)$. Consider an orbit of length $l$ in $\operatorname{Fix}\left(f_{1}^{k}\right)$. Then $l \mid k$ and the orbit represents an orbit $[\alpha]^{l} \in \mathcal{I E O} \mathcal{R}\left(f^{l}\right)$. Moreover since $f$ is linear and $L\left(f^{l}\right) \neq 0$, \# $\operatorname{Fix}\left(f_{1}^{l}\right)=N\left(f^{l}\right)$ so no other points represent $[\alpha]^{l}$. Thus each orbit of points in $\operatorname{Fix}\left(f_{1}^{k}\right)$ represents an orbit in $\mathcal{I E O} \mathcal{R}$ and no other point represents this orbit. Now Lemma 4.1 gives the equality

$$
\operatorname{Fix}\left(f_{1}^{k}\right) \geq \sum_{l \mid k} \mathcal{I E O R}\left(f^{l}\right) \cdot l=N F_{k}(f) .
$$

\section{REFERENCES}

[1] I.K. Babenko And S.A. Bogatyi, The behavior of the index of periodic points under iterations of a mapping, Math. USSR Izv. 38 (1992), 1-26.

[2] Sc R. Brooks, R. Brown, J. Pak and D. Taylor, Nielsen numbers of maps of tori, Proc. Amer. Math. Soc. 52 (1975), 398-400.

[3] R. F. Brown, The Lefschetz Fixed Point Theorem, Glenview, New York, 1971.

[4] S.N. Chow, J. Mallet-Paret and J.A. Yorke, A periodic point index which is a bifurcation invariant, Geometric dynamics (Rio de Janeiro, 1981), 109-131, Springer Lecture Notes in Math. 1007, Berlin 1983.

[5] A. Dold, Fixed point indices of iterated maps, Invent. Math. 74 (1983), 419-435.

[6] G. Graff and J. JeZIerski, Minimal number of periodic points for $C^{1}$ self-maps of compact simply-connected manifolds, Forum Math. 21 (2009), no. 3, 491-509.

[7] _ Minimizing the number of periodic points for smooth maps. Non-simply connected case, Topology Appl. 158 (2011), no. 3, 276-290.

[8] G. Graff, J. Jezierski and P. Nowak-Przygodzki, Fixed point indices of iterated smooth maps in arbitrary dimension J. Differential Equations 251 (2011), no. 6, 15261548.

[9] G. Graff and P. Nowak-Przygodzki, Fixed point indices of iterations of $C^{1}$ maps in $\mathbb{R}^{3}$, Discrete Cont. Dyn. Systems 16 (2006), no. 4, 843-856.

[10] B. Halpern, Periodic points on tori, Pacific J. Math. 83 (1979), no. 1, 117-133.

[11] Ph. Heath and E. Keppelmann, Fibre techniques in Nielsen periodic point theory on nil and solvmanifolds I, Topology Appl. 76 (1997), no. 3, 217-247.

[12] _ Fibre techniques in Nielsen periodic point theory on nil and solvmanifolds II, Topology Appl. 106 (2000), no. 2, 149-167.

[13] J. Jezierski, Wecken's theorem for periodic points in dimension at least 3, Topology Appl. 153 (2006), no. 11, 1825-1837.

[14] _ The least number, of $n$-periodic points of a self-map of a solvmanifold, can be realised by a smooth map, Topology Appl. 158 (2011), no. 9, 1113-1120.

[15] _ Least number of periodic points of self-maps of Lie groups, Acta Math. Sinica 30 (2014), no. 9, 1477-1494.

[16] J. Jezierski and W. Marzantowicz, Homotopy methods in topological fixed and periodic points theory, Topological Fixed Point Theory and Its Applications, Vol. 3. Springer, Dordrecht, 2006. xii+319 pp.

[17] B.J. Jiang, Lectures on the Nielsen Fixed Point Theory, Contemp. Math. 14, Amer. Math. Soc., Providence 1983.

[18] _ Fixed point classes from a differential viewpoint, in: Lecture Notes in Math. 886, Springer, (1981), 163-170. 
[19] M. Shub And P. Sullivan, A remark on the Lefschetz fixed point formula for differentiable maps, Topology 13 (1974), 189-191.

[20] C.Y. You, The least number of periodic points on tori Adv. in Math. (China) 24 (1995), no. $2,155-160$

Manuscript received July 7, 2014

accepted January 18, 2015

JERZY JEZIERSKI

Department of Applied Informatics and Mathematics

Warsaw University of Life Sciences

Nowoursynowska 159

02-757 Warszawa, POLAND

E-mail address: jerzy_jezierski@sggw.pl

TMNA : Volume $47-2016-\mathrm{N}^{\mathrm{O}} 1$ 